\title{
CONVEX FUNCTIONS AND SCHWARZ DERIVATIVES
}

\author{
HAJRUDIN FEJZIĆ
}

(Communicated by Andrew Bruckner)

\begin{abstract}
If the lower Schwarz derivate of a continuous function is nonnegative, then it is convex. The main result in this paper is that if the lower Schwarz derivate of a measurable function $f$ is nonnegative, then there is a dense open set with $f$ convex on each component.
\end{abstract}

We begin by recalling the definition of a lower Schwarz derivate.

Definition 1. Let $f$ be a function defined on an open interval $I$, and $x \in I$. For each $h>0$ so small that $x+h$ and $x-h$ both lie in $I$ define

$$
\Delta_{2} f(x, h)=(f(x+h)+f(x-h)-2 f(x)) / h^{2},
$$

and let

$$
L D_{2} f(x)=\liminf _{h \rightarrow 0^{+}} \Delta_{2} f(x, h) .
$$

$L D_{2}$ is called the lower Schwarz derivate.

It is well known and easy to prove that if $f$ is continuous on an open interval $I$ and if the lower Schwarz derivate (even the upper Schwarz derivate) is nonnegative, then $f$ is convex on $I$. For this and some other simple properties of convex functions the reader is referred to [3].

A natural question to ask is whether $L D_{2} f(x) \geq 0$ still implies convexity on $I$ when continuity is replaced by weaker conditions. The first major result in this direction is the following result of C. Weil. (See [2].)

Theorem 2. Let $I$ be an open interval and $f$ a Baire one, Darboux function defined on I. If $L D_{2} f(x) \geq 0$ for each $x \in I$, then $f$ is convex on $I$.

Later, Z. Buczolich proved the following theorem. (See [1].)

Theorem 3. Let $I$ be an open interval and $f$ a measurable function defined on I such that $L D_{2} f(x) \geq 0$ for each $x \in I$. If $f$ is a sum of a continuous function and Darboux function, then $f$ is convex on $I$.

Weil's proof of Theorem 2 is lengthy and very complicated. Buczolich's proof of Theorem 3 shows how to modify Weil's proof for measurable functions and it is based on the following property of measurable functions with positive Schwarz derivates.

Received by the editors September 15, 1993 and, in revised form, December 13, 1993.

1991 Mathematics Subject Classification. Primary 26A21, 26A48, 26A51. 
Lemma 4. Let $f$ be a real-valued measurable function defined on an open interval $I$ such that $D L_{2} f(x)>0$ for every $x \in I$. If $f$ is bounded by $B$ on a dense set of some open interval $K$, then $f$ is bounded by $B$ on all of $K$.

We give a proof of this lemma that differs from the proof in [1]. For the proof of Lemma 4, Lemma 5, Theorem 6, and Corollary 7 below, we define for each $x \in I$

$$
\delta(x)=\sup \{\gamma>0: f(x+h)+f(x-h)-2 f(x)>0 \text { for all } 0<h<\gamma\} .
$$

For $n \in N$, let

$$
A_{n}=\{x: \delta(x) \geq 1 / n \text { and } f(x) \leq n\} .
$$

If $D L_{2} f(x)>0$ for all $x \in I$, then $\delta(x)$ is well defined and $\bigcup_{n=1}^{\infty} A_{n}=I$.

Proof of Lemma 4. Let $A=\{x \in K: f(x) \leq B\}$. If $A$ has full measure in $K$ let $x$ be any point in $K$. Then one can find $0<h<\delta(x)$ such that both $x-h$ and $x+h$ are in $A$, which implies that $f(x) \leq B$, and we are done.

If $A$ does not have full measure, then for some positive rational number $m$,

$$
C_{m}=\{x \in K: B+m<f(x)<B+2 m\}
$$

has positive measure, and then for some $n, C_{m} \cap A_{n}$ has positive outer measure. Then there is an interval $[a, b] \subset K$, such that $b-a<1 / n$ and where the outer measure of $C_{m} \cap A_{n}$ is bigger than $\frac{5}{6}(b-a)$. Let $J_{0}=\left[a, a+\frac{b-a}{3}\right]$. Then $D=J_{0} \cap C_{m} \cap A_{n}$ has outer measure bigger than $\frac{1}{6}(b-a)$. Choose a point $x \in A$ such that $x<a$ and $a-x<(b-a) / 3$. Then the set $2 D-x$ has outer measure bigger than $\frac{1}{3}(b-a)$. If $d \in D$, then $f(x)-2 f(d)+f(2 d-x)>$ 0 . Hence $f(2 d-x)>2(B+m)-B=B+2 m$. Then the disjoint sets $f^{-1}(B+2 m, \infty) \cap[a, b]$ and $f^{-1}(-\infty, B+2 m) \cap[a, b]$ are measurable, and since $2 D-x \subset f^{-1}(B+2 m, \infty) \cap[a, b]$ and $D \subset f^{-1}(-\infty, B+2 m) \cap[a, b]$, their measures are bigger than $\frac{5}{6}(b-a)$ and $\frac{1}{3}(b-a)$ respectively, a contradiction.

That the same conclusion holds if measurability in Lemma 4 is replaced by Baire property is the statement of Lemma 5.

Lemma 5. Let $f$ be a real-valued function with the property of Baire defined on an open interval $I$ such that $D L_{2} f(x)>0$ for every $x \in I$. If $f$ is bounded by $B$ on a dense set of some open interval $K$, then $f$ is bounded by $B$ on all of $K$.

Proof. Let $A=\{x \in K: f(x) \leq B\}$. If $A$ is a residual set in $K$, then for any $x \in K$ we can pick $0<h<\delta(x)$ such that both $x-h$ and $x+h$ are in $A$, which implies that $f(x) \leq B$, and we are done.

If $A$ is not residual, then for some positive rational number $m$,

$$
C_{m}=\{x \in K: B+m<f(x)<B+2 m\} \neq \varnothing
$$

is second category in $K$. Since $f$ has the property of Baire, there is a subinterval $[c, d] \subset K$ such that $C_{m}$ is residual in $[c, d]$. By the Baire Category Theorem there is an interval $[a, b] \subset\left[c, \frac{c+d}{3}\right]$ such that for some integer $n$, $C_{m} \cap A_{n}$ is second category in $[a, b]$. Without loss of generality we may assume that $b-a<\frac{1}{2 n}$. Since $A$ is dense in $K$, one can find a point $x \in A$ such that 
$x<a$ and $a-x<\frac{1}{2 n}$. The set $2\left(C_{m} \cap A_{n} \cap[a, b]\right)-x$ is second category in $[c, d]$. Therefore the intersection $\left(2\left(C_{m} \cap A_{n} \cap[a, b]\right)-x\right) \cap\left(C_{m} \cap[c, d]\right)$ is nonempty. Let $y$ be from the intersection. Then there is a $z \in C_{m} \cap A_{n}$ such that $z=\frac{x+y}{2}$. Hence $f(x)-2 f(z)+f(y)>0$. It follows that $f(x)>$ $2 f(z)-f(y)>2(B+m)-B+2 m=B$, which contradicts $x \in A$.

The main result of the paper is the following theorem.

Theorem 6. Let I be an open interval. If $f$ is a measurable function (or with the property of Baire) defined on I such that $D L_{2} f(x) \geq 0$ for every $x \in I$, then there is an open dense set $G$ in $I$ such that $f$ is convex on every component of $G$. Conversely, if $G$ is an open dense set in $I$, then there is a measurable function $f$ (with the property of Baire) defined on I such that $D L_{2} f(x) \geq 0$ on $I$ and if $J \subset I$ is any interval on which $f$ is convex, then $J \subset G$.

Proof. In the first part of Theorem 6 it suffices to prove that for a measurable function (or with the property of Baire) with $D L_{2} f(x)>0$ on $I$ there is a dense open set $G$ such that $f$ is convex on each component of $G$. To see this, consider $F(x)=f(x)+x^{2}$. Then $D L_{2} F(x)>0$ on $I$. By proving the theorem for $F$ we actually get that $F$ is continuous on every component of $G$. Then $f$ is continuous on every component of $G$ and since $D L_{2} f(x) \geq 0$, $f$ is convex on every component of $G$. So, we assume $D L_{2} f(x)>0$ for all $x \in I$.

For each $a, b \in I$, define a function $g$ by subtracting from $f$ the line connecting $(a, f(a))$ and $(b, f(b))$, i.e.,

$$
g(x)=f(x)-\frac{x-a}{b-a} f(b)-\frac{b-x}{b-a} f(a) .
$$

Then $g(a)=g(b)=0$ and

(3) $g(x+h)+g(x-h)-2 g(x)=f(x+h)+f(x-h)-2 f(x)$ for all $x \in I$.

Let $K \subset I$ be an interval. Since $K \subset \bigcup_{n=1}^{\infty} A_{n}$, by the Baire Category Theorem there is an integer $n$ and an interval $J \subset K$ of length less than $1 / n$ so that $A_{n}$ is dense in $J$. Let $[a, b] \subset J, g$ be as in (2), and $c=$ $\sup \{g(x): x \in[a, b]\}$. By Lemma 4 (Lemma 5), $c<+\infty$. Suppose that $c>0$. This will lead to a contradiction as follows.

First we show that, there is a sequence $\left\{y_{k}\right\} \subset A_{n} \cap(a, b)$ such that $g\left(y_{k}\right)$ converges to $c$. Suppose not. Then there is an $\varepsilon>0$ such that $\sup \{g(y): y \in$ $\left.A_{n}\right\}<c-\varepsilon$. Pick an $x \in(a, b)$ such that $g(x)>c-\varepsilon / 2$. Since $A_{n}$ is dense in $[a, b]$, there is a $y \in A_{n} \cap(a, b)$ such that $|y-x|<\delta(x)$ and $2 x-y \in(a, b)$. Therefore, by (1) and (3) $g(x)<1 / 2(g(y)+g(2 x-y))<1 / 2(c-\varepsilon+c)=c-\varepsilon / 2$, a contradiction.

Since $\left\{y_{k}\right\} \subset[a, b]$, we may assume it is convergent. Let $y \in[a, b]$ be the limit point of the sequence. If $g(y)=c>0$, then $y \in(a, b)$, and if $z \in(a, b)$ is such that $|z-y|<\delta(y)$ and $2 y-z \in(a, b)$, then (1) and (3) imply that either $g(z)$ or $g(2 z-y)$ has to be strictly bigger than $g(y)$, which violates the choice of $c$. Therefore $g(y)<c$. Now pick $k$ so that $c-g\left(y_{k}\right)<g\left(y_{k}\right)-g(y)$ and that $2 y_{k}-y \in(a, b)$. Then, again by (3), and the fact that $\delta\left(y_{k}\right) \geq 1 / n$, we have $g\left(2 y_{k}-y\right)>2 g\left(y_{k}\right)-g(y)>c+g(y)-g(y)=c$, a contradiction. Therefore,

$$
\text { if } x \in[a, b] \subset J \text {, then } g(x) \leq 0 \text {. }
$$


This implies that for every $x \in[a, b], f(x)$ is below the line segment connecting the points $(a, f(a))$ and $(b, f(b))$. Since $[a, b]$ was an arbitrary interval in $J$, we have that $f$ is convex (and thus continuous) on $J$. Let $G \subset I$ be the union of all open intervals on which $f$ is convex. If $(a, b)$ is a component interval of $G$, then $f$ is continuous on $(a, b)$, and since $D L_{2} f(x)>0$ for all $x \in(a, b)$, it is convex there. Finally, since $K$ was an arbitrary open interval in $I, G$ is dense in $I$.

Conversely, let $G$ be an open dense subset of $I$. Then

$$
f(x)= \begin{cases}x^{2} & \text { if } x \in G \\ 0 & \text { otherwise }\end{cases}
$$

satisfies the conditions of the theorem.

The following corollary is a generalization of Theorem 3.

Corollary 7. Let $I$ be an open interval and $f$ a measurable function (or with the property of Baire) defined on $I$ with $D L_{2} f(x) \geq 0$ for each $x \in I$. Let $G$ be the dense open set from Theorem 6 . If $f$ is continuous on the closure of every component of $G$, then $f$ is convex on $I$.

Proof. Let $C=I \backslash G$. Note that $G$ is a maximal open set such that $f$ is convex on every component of $G$. If $b \in C^{\circ}$ is an isolated point, then there are intervals $(a, b)$ and $(b, d)$ in $G$. Then $f$ is continuous at $b$ and hence $f$ is convex on $(a, d)$. Therefore $C$ is perfect.

If $C \neq \varnothing$, then by the Baire Category Theorem there is an integer $n$ and an open interval $J \subset I$ of length less than $1 / n$ so that $A_{n}$ is dense in $C \cap J \neq 0$. Let $[a, b] \subset J$ and $g$ be as in (2). Our goal is to show that if $x \in[a, b]$, then $g(x) \leq 0$. If $[a, b] \subset G$, then, since in that case $g$ is convex on $[a, b]$, for every $x \in[a, b]$ we have that $g(x) \leq 0$. So we may assume $[a, b] \cap A_{n} \neq \varnothing$. Let $c=\sup \left\{g(x): x \in A_{n} \cap[a, b]\right\}$. Let $(d, e)$ be a component of $G \cap[a, b]$. If $e=b$ and $g(d) \leq g(e)$, then by convexity $g(x) \leq g(b)=0$ for all $x \in$ $[d, e]$. If $e<b$ and $g(d) \leq g(e)$, then for $x \in(e, 2 e-d), x-e<\delta(e)$, $g(2 e-x)-2 g(e)+g(x)>0$. Since $d<2 e-x<e$, it follows that $g(x)>g(e)$. In particular, this is true for some $x \in A_{n}$ since $A_{n}$ is dense in $C \cap[a, b]$. We conclude that if $y \in[d, e]$, then $g(y) \leq \max (g(x), 0) \leq|c|$. A similar argument holds if $g(d) \geq g(e)$.

Now pick any $x \in[a, b]$. Then for some $0<h<\delta(x)$, both $x-h$ and $x+h$ are in $G \cap[a, b]$. Since $g(x+h)$ and $g(x-h)$ are bounded by $|c|$, it follows that $g(x) \leq|c|$.

Assume $c>0$. Let $\left\{y_{k}\right\} \subset[a, b] \cap A_{n}$ be a convergent sequence such that $g\left(y_{k}\right) \rightarrow c$ and let $y \in[a, b]$ be the limit point of $\left\{y_{k}\right\}$. Proceeding as in the proof of (4), we get $g(y)<c$ which gives a nearby point $z$ such that $g(z)>c$, which is a contradiction.

Therefore $c=0$, and hence if $x \in[a, b]$, then $g(x) \leq 0$. As in the proof of Theorem 6 , this implies $f$ never goes above the line segment joining $(a, f(a))$ and $(b, f(b))$. Since $[a, b]$ is arbitrary, $f$ is convex on $J$, contradicting the maximality of $G$. Hence $C=\varnothing$.

\section{ACKNOWLEDGMENTS}

The author would like to thank Professor Chris Freiling and Professor Dan Rinne for the generous help they offered during the preparation of this paper. 


\section{REFERENCES}

1. Z. Buczolich, Convexity and symmetric derivates of measurable functions, Real Anal. Exchange 16 (1990-91), 187-196.

2. C. E. Weil, Monotonicity, convexity and symmetric derivates, Trans. Amer. Math. Soc. 221 (1976), 225-237.

3. A. Zygmund, Trigonometric series, Cambridge Univ. Press, Cambridge and New York, 1990.

Department of Mathematics, California State University, San Bernardino, CaliforNIA 92407

E-mail address: hfejzic@iley.csusb.edu 